PSS PROCEEDINGS

\title{
The turnaround radius as a probe of dark energy and modified gravity
}

\author{
Valerio Faraoni* \\ Department of Physics and Astronomy, Bishop's University, Sherbrooke, Québec, Canada \\ J1M $1 Z 7$ \\ E-mail: vfaraoni@ubishops.ca
}

The turnaround radius in cosmology is investigated as a possible probe of dark energy and modified gravity scenarios.

EPS-HEP 2017, European Physical Society conference on High Energy Physics 5-12 July 2017

Venice, Italy

${ }^{*}$ Speaker. 


\section{Introduction}

We propose a novel approach to the problem of the turnaround radius of a large structure in an accelerating universe. This new approach is based on the Hawking quasilocal energy of General Relativity (GR) and is part of a broader program aiming at applying this formal concept to cosmology. Thus far, the quasilocal energy has been applied to Newtonian $N$-body simulations of large scale structure formation [1], and to the decade-old problem of lensing by the cosmological constant [2].

\section{Turnaround radius with Hawking mass in GR}

Consider the present accelerated era of the universe and the largest bound objects in the sky. It was suggested recently that the turnaround radius is a possible probe of dark energy or modified gravity scenarios $[3,4,5,6]$ but the concept of turnaround radius is older [7].

In an accelerated FLRW universe with one spherical inhomogeneity, massive test particles with zero radial initial velocity cannot collapse if they lie outside a critical radius $R_{c}$ (turnaround radius $),{ }^{1}$ but they only expand. Dust shells with $R<R_{c}$ collapse but, if you are in geodesic motion outside $R_{c}$, you will never fall back.

Over the years, the turnaround radius has been studied in exact solutions of the Einstein equations, including the Schwarzschild-de Sitter, Lemaître-Tolman-Bondi (LTB), and McVittie spacetimes. In the heuristic Schwarzschild-de Sitter space with line element

$$
d s^{2}=-\left(1-\frac{2 M}{R}-H^{2} R^{2}\right) d t^{2}+\frac{d R^{2}}{1-\frac{2 M}{R}-H^{2} R^{2}}+R^{2} d \Omega_{(2)}^{2}
$$

where $H=\sqrt{\Lambda / 3}$, the turnaround radius

$$
R_{c}=\left(\frac{3 G M}{\Lambda}\right)^{1 / 3}
$$

is obtained by setting to zero the acceleration along radial timelike geodesics. In LTB models with dust and a cosmological constant $\Lambda$

$$
d s^{2}=-d t^{2}+\frac{R^{\prime}(t, r)}{1+f(r)} d r^{2}+R^{2}(t, r) d \Omega_{(2)}^{2}
$$

(where ${ }^{\prime} \equiv d / d r$ and $f(r)$ is related to the initial density profile), radial timelike geodesics obey

$$
\ddot{R}=-\frac{G \mathscr{M}(r)}{R^{2}}+\frac{\Lambda R}{3}
$$

and the authors of [5] obtain the turnaround radius

$$
R_{c}=\left(\frac{3 G \mathscr{M}\left(r_{c}\right)}{\Lambda}\right)^{1 / 3},
$$

\footnotetext{
${ }^{1} R$ denotes the areal radius, a geometric quantity which is covariant and gauge-invariant.
} 
where

$$
\mathscr{M}(r) \equiv \int_{0}^{R} d R R^{2} \rho
$$

is the Lemaitre mass.

While exact solutions of the Einstein equations are toy models, a realistic description of the universe uses the perturbed Friedmann-Lemaître-Robertson-Walker (FLRW) line element

$$
d s^{2}=a^{2}(\eta)\left[-(1+2 \phi) d \eta^{2}+(1-2 \phi)\left(d r^{2}+r^{2} d \Omega_{(2)}^{2}\right)\right] .
$$

The timelike radial geodesics obey [5]

$$
\ddot{R}=\frac{\ddot{a}}{a}-\frac{G \mathscr{M}(r)}{R^{2}},
$$

and it is suggested implicitly in [5] that $\mathscr{M}(r)$ is the Lemaitre mass (2.6), from which one obtains the turnaround radius [5]

$$
R_{c}=\left(\frac{3 \mathscr{M}}{4(3 w+1) \pi \rho_{D E}}\right)^{1 / 3} .
$$

Two questions arise: the issue of the gauge-invariance of this result and, more important, it is not clear what the "mass contained in a sphere of radius $R$ " is. Should it include the dark energy density $\rho_{D E}$ ? If not, why? Should it include only $\rho_{\text {perturbation }}$ ? If so, why? The Hawking-Hayward quasilocal energy $M_{H H}$ of GR, which includes all energy forms, is suitable for answering both questions. In spherical symmetry it reduces to the Misner-Sharp-Hernandez mass [8]. We assume that GR is valid, we restrict to first order in the metric perturbation which (as customary in the literature on the turnaround radius) is assumed to be spherically symmetric $(\phi=\phi(r))$, and we assume that the background universe is a spatially flat FLRW one accelerated by dark energy with density $\rho_{D E}$ and pressure $P_{D E}=w \rho_{D E}$.

The physical Hawking-Hayward quasilocal energy [9], based on the idea that the total mass in a region bounded by a 2 -surface $S$ is measured by its effect on null geodesics at $S$, is defined as follows [9]. Let $S$ be a closed spacelike orientable 2-surface, $\mathscr{R}$ the induced Ricci scalar on $S, \theta_{( \pm)}$ the expansions of outgoing/ingoing null geodesic congruences from $S, \sigma_{a b}^{( \pm)}$their shear tensors, $\omega^{a}$ the anholonomicity (i.e., the projection on $S$ of the commutator of the null normal vectors to $S$ ), $\mu$ the volume 2 -form on $S$, and $A$ the area of $S$. Then,

$$
M_{H H} \equiv \frac{1}{8 \pi} \sqrt{\frac{A}{16 \pi}} \int_{S} \mu\left(\mathscr{R}+\theta_{(+)} \theta_{(-)}-\frac{1}{2} \sigma_{a b}^{(+)} \sigma_{(-)}^{a b}-2 \omega_{a} \omega^{a}\right) .
$$

By computing this quantity for the line element (2.7) we find that, to first order, it decomposes as [10]

$$
\tilde{M}_{H H}=\underbrace{\Omega M_{H H}-\frac{R \Omega_{, \eta}}{4 \pi} \frac{\Omega_{, \eta}}{\Omega} \int_{S} \mu \phi_{N}}_{\text {local }}+\underbrace{\frac{R^{3}}{2} \frac{\Omega_{, \eta}^{2}}{\Omega}}_{\text {cosmological }} .
$$

In spherical symmetry this decomposition reduces to $[1,11,2]$

$$
M_{H H}=m a+\frac{H^{2} R^{3}}{2}(1-\phi) \simeq m a+\frac{H^{2} R^{3}}{2},
$$


where

$$
m \equiv \frac{1}{4 \pi} \int d^{3} \vec{x} \nabla^{2} \phi
$$

is the Newtonian mass of the perturbation. Our new criterion identifies the turnaround radius by equating the two contributions to the quasilocal mass, which yields

$$
R_{c}(t)=\left(\frac{2 m a}{H^{2}}\right)^{1 / 3}
$$

The Friedmann equation $H^{2}=8 \pi G \rho_{D E} / 3$ then gives $R_{c}(t)=\left(\frac{3 m a}{4 \pi \rho_{D E}}\right)^{1 / 3}$. If, in addition, the equation of state parameter $w$ of the dark energy is constant, one obtains $R_{c}=\left(\frac{3 m a}{4 \pi \rho_{0}}\right)^{1 / 3} a^{(3 w+4) / 3}$. For comparison, the ratio of the turnaround radius (2.14) to that of [5] is

$$
\frac{R_{c}}{R_{c}^{(P T T)}}=\left(\frac{|3 w+1|}{2}\right)^{1 / 3} \approx 1 \quad \text { if } w \approx-1 .
$$

Although the difference is numerically small, in our approach there is no ambiguity in the concept of "mass inside a sphere of radius $R_{c}$ " and the description is gauge-invariant. We have, therefore, a rigorous derivation of turnaround radius.

The turnaround radius may provide a way to constrain the equation of state parameter $w$ of dark energy. One can express it as a function of redshift $R_{c}(z)$ and obtain [5]

$$
\int d z \frac{w(z)+1}{z+1}=\ln \left[\left(\frac{3 m a}{4 \pi \rho}\right)^{1 / 3} \frac{1}{R(z)}\right] .
$$

If $w=$ const., this expression reduces to

$$
w=-1+\frac{\ln \left[\left(\frac{3 m a}{4 \pi \rho_{0}}\right)^{1 / 3} \frac{1}{R_{c}(z)}\right]}{\ln (z+1)}
$$

which, in principle, allows one to constrain $w$ if $m a$ and $R_{c}$ are known.

\section{Turnaround radius in scalar-tensor gravity}

Consider now scalar-tensor gravity. Because there is no universally accepted notion of quasilocal energy in this class of theories (see, however, the proposals [12]), we proceed with the usual (gauge-dependent) method of setting to zero the acceleration along radial timelike geodesics. The perturbed FLRW spacetime is now described by the line element

$$
d s^{2}=a^{2}(\eta)\left[-(1+2 \psi) d \eta^{2}+(1-2 \phi)\left(d r^{2}+r^{2} d \Omega_{(2)}^{2}\right)\right]
$$

with two perturbation potentials $\phi=\phi(r), \psi=\psi(r)$. The radial timelike geodesics are described by

$$
\begin{aligned}
& \frac{d u^{0}}{d \tau}+\frac{a_{\eta}}{a}\left(u^{0}\right)^{2}+2 \psi^{\prime} u^{0} u^{1}+\frac{a_{\eta}}{a}(1-2 \phi-2 \psi)\left(u^{1}\right)^{2}=0 \\
& \frac{d u^{1}}{d \tau}+\psi^{\prime}\left(u^{0}\right)^{2}+\frac{2 a_{\eta}}{a} u^{0} u^{1}-\phi^{\prime}\left(u^{1}\right)^{2}=0,
\end{aligned}
$$


where $u_{c} u^{c}=-1$. The areal radius is $R(t, r)=\operatorname{ar} \sqrt{1-2 \phi} \simeq \operatorname{ar}(1-\phi)$. Manipulations yield [13]

$$
\frac{d^{2} R}{d t^{2}}=\left[\ddot{a} r+\frac{\dot{a} u^{1}}{a u^{0}}+\frac{1}{a u^{0}} \frac{d}{d \tau}\left(\frac{u^{1}}{u^{0}}\right)\right](1-\phi) .
$$

The criterion $d^{2} R / d t^{2}=0$ locating the (unique) turnaround radius yields

$$
\ddot{a} r-\frac{\psi^{\prime}}{a}=0
$$

or, in terms of the areal turnaround radius, $R_{c}=a(t) r_{c}\left[1-\phi\left(r_{c}\right)\right]$ or again, using the gravitational $\operatorname{slip} \xi \equiv(\phi-\psi) / \phi$,

$$
\ddot{a} R_{c}\left(1+\phi_{c}\right)-\phi_{c}^{\prime}\left(1-\xi_{c}\right)+\phi_{c} \xi_{c}^{\prime}=0 .
$$

\section{Conclusions}

The turnaround radius constitutes a potential probe of gravity and/or of the $\Lambda \mathrm{CDM}$ model. For spherical perturbations of a FLRW universe in GR, the (covariant) Hawking-Hayward/MisnerSharp-Hernandez mass $M_{H H}$ splits, to first order, into a local part and a cosmological part. This covariant splitting allows a rigorous derivation of the turnaround radius $R_{C}$ and provides a small numerical correction to the value of $R_{c}$ in the literature, but a much needed clarification of "mass contained in a sphere of radius $R$ ".

In modified (scalar-tensor or $f(R)$ ) gravity, there is no universally accepted notion of quasilocal mass $M_{H H}$. In this case, the usual criterion $\ddot{R}=0$ along radial timelike geodesics yields an expression of $R_{C}$ in this class of theories.

Is the turnaround radius important? A recent claim by astronomers [6] that the upper bound set by GR on $R_{c}$ is exceeded by far in the galaxy group NGC 5353/4 has been taken back due to the previously underestimated error introduced by the non-sphericity of the system [14]. However, the verdict on the usefulness of the turnaround radius as a cosmological probe is still out and requires further and careful study.

\section{References}

[1] V. Faraoni, M. Lapierre-Léonard, and A. Prain, Phys. Rev. D 92 (2015) 023511. DOI: 10.1088/1475-7516/2015/10/013

[2] V. Faraoni and M. Lapierre-Léonard, Phys. Rev. D 95 (2017) 023509. DOI: 10.1103/PhysRevD.95.023509.

[3] Z. Roupas, M. Axenides, G. Georgiou, and E.N. Saridakis, Phys. Rev. D 89 (2014) 083002, DOI: 10.1103/PhysRevD.89.083002; D. Tanoglidis, V. Pavlidou, and T.N. Tomaras, JCAP 1512 (2015) 060, DOI: $10.1088 / 1475-7516 / 2015 / 12 / 060$.

[4] V. Pavlidou and T.N. Tomaras, JCAP 1409 (2014) 020, 10.1088/1475-7516/2014/09/020.

[5] V. Pavlidou, N. Tetradis, and T.N. Tomaras, JCAP 1405 (2014) 017, DOI: 10.1088/1475-7516/2014/05/017

[6] J. Lee, S. Kim, and S.G. Rey, Astrophys. J. 815 (2015) 43, DOI: 10.1088/0004-637X/815/1/43; J. Lee, Astrophys. J. 832 (2016) 123, DOI: 10.3847/0004-637X/832/2/123. 
[7] J.M. Souriau, Un modèle d'univers confronté aux observations, in Dynamics and Processes, Proceedings of the Third Encounter in Mathematics and Physics, Bielefeld, Germany, Nov. 30-Dec. 4, 1981, Lecture Notes in Mathematics vol. 1031, P. Blanchard, W. Streit eds., Springer-Verlag, Berlin (1981), p. 114-160; Z. Stuchlik, Bull. Astronomical Institutes of Czechoslovakia 34 (1983) 129; Z. Stuchlik and S. Hledik, Phys. Rev. D 60 (1999) 044006, DOI: 10.1103/PhysRevD.60.044006; Z. Stuchlik, P. Slany, and S. Hledik, Astron. Astrophys. 363 (2000) 425; Z. Stuchlik, Mod. Phys. Lett. A 20 (2005) 561, DOI: 10.1142/S0217732305016865; M. Mizony and M. Lachiéze-Rey, Astron. Astrophys. 434 (2005) 45, DOI: 10.1051/0004-6361:20042195; Z. Stuchlik, and J. Schee, JCAP 9 (2011) 018, DOI: 10.1088/1475-7516/2011/09/018; B.C. Nolan, Class. Quantum Grav. 31 (2014) 235008, DOI: 10.1088/0264-9381/31/23/235008; M. Blau and B. Rollier, Class. Quantum Grav. 25 (2008) 105004, DOI: 10.1088/0264-9381/25/10/105004.

[8] C.W. Misner and D.H. Sharp, Phys. Rev. 136 (1964) B571, DOI: 10.1103/PhysRev.136.B571; W.C. Hernandez and C.W. Misner, Astrophys. J. 143 (1966) 452, DOI: 10.1086/148525.

[9] S.W. Hawking, J. Math. Phys. 9 (1968) 598, DOI: 10.1063/1.1664615; S.A. Hayward, Phys. Rev. D 49 (1994) 831, DOI: 10.1103/PhysRevD.49.831.

[10] A. Prain, V. Vitagliano, V. Faraoni, and M. Lapierre-Léonard, CQG 33 (2016) 145008, DOI: 10.1088/0264-9381/33/14/145008.

[11] V. Faraoni, M. Lapierre-Léonard, and A. Prain, JCAP 10 (2015) 013, DOI: $10.1088 / 1475-7516 / 2015 / 10 / 013$.

[12] R.G. Cai, L.M. Cao, Y.P. Hu, and N. Ohta, Phys. Rev. D 80 (2009) 104016, DOI: 10.1103/PhysRevD.80.104016; R.G. Cai, L.M. Cao, Y.P. Hu, and S.P. Kim, Phys. Rev. D 78 (2008) 124012, DOI: 10.1103/PhysRevD.78.124012; H. Zhang, Y. Hu, and X.-Z. Li, Phys. Rev. D 90 (2014) 024062, DOI: 10.1103/PhysRevD.90.024062; S.-F. Wu, B. Wang, and G.-H. Yang, Nucl. Phys. B 799 (2008) 330, DOI: 0.1016/j.nuclphysb.2008.01.013; G. Cognola, O. Gorbunova, L. Sebastiani, and S. Zerbini, Phys. Rev. D 84 (2011) 023515, DOI: 10.1103/PhysRevD.84.023515; V. Faraoni, Class. Quantum Grav. 33 (2015) 015007, DOI: 10.1088/0264-9381/33/1/015007; F. Hammad, Class. Quantum Grav. 33 (2016) 235016, DOI: 10.1088/0264-9381/33/23/235016.

[13] V. Faraoni, Phys. Dark Univ. 11 (2016) 11. DOI: 10.1016/j.dark.2015.11.001.

[14] J. Lee and G. Yepes, Astrophys. J. 832 (2016) 185. DOI: 10.3847/0004-637X/832/2/185. 